\title{
Characteristics of anisometropic suppression: Simple reaction time measurements
}

\author{
MICHAEL J. PIANTA and MICHAEL KALLONIATIS \\ University of Melbourne, Melbourne, Victoria, Australia
}

\begin{abstract}
The characteristics of artificially induced anisometropic suppression were investigated in observers with normal and abnormal binocular vision (anisometropic amblyopia) by using a simple reaction time paradigm. Reaction time was measured as a function of stimulus intensity for various stimulus durations. For all conditions, the reaction time increased as stimulus intensity decreased toward threshold. We found that traditional techniques for modeling this trend were inadequate, so we developed a simple visuogram method for comparing these functions. Using this technique, reaction time versus intensity functions are shown to be shape-invariant for all conditions examined. This means that, although reaction times are longer during induced anisometropic suppression or in anisometropic amblyopia, they are the same if contrast is normalized to equate threshold. The shapeinvariant nature of these functions is also consistent with the notion that a single mechanism mediates detection under these conditions. Temporal summation was investigated at both threshold (method of limits) and suprathreshold (criterion reaction time) levels. Again, because of shape invariance, the suprathreshold results mirror the threshold results. The critical duration (the duration at the intersection of the complete summation and zero summation regions) is not affected by any of the conditions. However, the critical intensity (the intensity for the zero summation region) is higher for the amblyopic eyes, as compared with the normal or nonamblyopic eyes. Induced anisometropic suppression always increases the critical intensity, with a smaller increase occurring for the amblyopic eyes. This suggests that amblyopic eyes do not have a need for strong suppression.
\end{abstract}

Suppression of all or part of the visual field of one eye is often associated with abnormal binocular vision. It occurs under binocular viewing conditions and is generally assumed (see, e.g., Burian \& von Noorden, 1974) to represent an adaptive mechanism that attempts to eliminate confusion (resulting from different images falling on corresponding retinal positions) and diplopia (resulting from the same images falling on disparate retinal positions). Suppression that occurs in abnormal binocular vision is usually called clinical suppression. However, suppression can also occur in individuals with normal binocular vision. Anisometropic suppression may be induced by defocusing the image in one eye relative to the other (Humphriss, 1959, 1960, 1963, 1982; Humphriss \& Woodruff, 1962; Schor, Landsman, \& Erickson, 1987; Simpson, 1991, 1992; Simpson, Smith, Harwerth, \& Kalloniatis, 1990; Wolfe \& Owens, 1979). Under these conditions, the amount of suppression depends on the level of anisometropia: With small levels, suppression does not occur; with intermediate lev-

This work was supported, in part, by National Health and Medical Research Council Grant 950607 and by a Special Initiatives Grant (from the University of Melbourne) to M.K. We thank three anonymous referces for their helpful suggestions on a previous version of this manuscript and associate editor R. P. O'Shea for valuable editorial comments. Correspondence concerning this article should be addressed to $M$. Kalloniatis, Department of Optometry and Vision Sciences, University of Melbourne, Melbourne, Victoria, 3052, Australia (e-mail: m.kalloniatis@ optometry.unimelb.edu.au). els, alternating periods of suppression and dominance are experienced; and, with large levels, suppression is constant (Simpson, 1991). Also, increasing the amount of induced anisometropia increases the size of the suppression scotoma (Simpson, 1991).

It has been hypothesized that chronic suppression is responsible for the development of amblyopia (see, e.g., DukeElder \& Wybar, 1973). It seems logical that anisometropic amblyopia (a developmental anomaly that occurs when the image in one eye is habitually defocused because of an imbalance in the refractive status between the eyes) may be a sensory consequence of anisometropic suppression, because both conditions develop in response to differential interocular image focus and both result in the functional loss of visual information from one eye. If this hypothesis were true, it would be reasonable to assume that common neural mechanisms underlie these conditions.

We have used a reaction time paradigm to compare the mechanisms responsible for the detection of different duration stimuli during induced anisometropic suppression and anisometropic amblyopia. Previous studies have used reaction times to differentiate the response properties of sustained and transient mechanisms in normal (Harwerth, Boltz, \& Smith, 1980; Harwerth \& Levi, 1978a) and abnormal binocular vision (Harwerth \& Levi, 1978b; Levi, Harwerth, \& Manny, 1979). These studies provide evidence that, in amblyopia, the sustained neural channels are more severely affected (i.e., have a higher intensity requirement) than are the transient channels. Different duration stimuli 


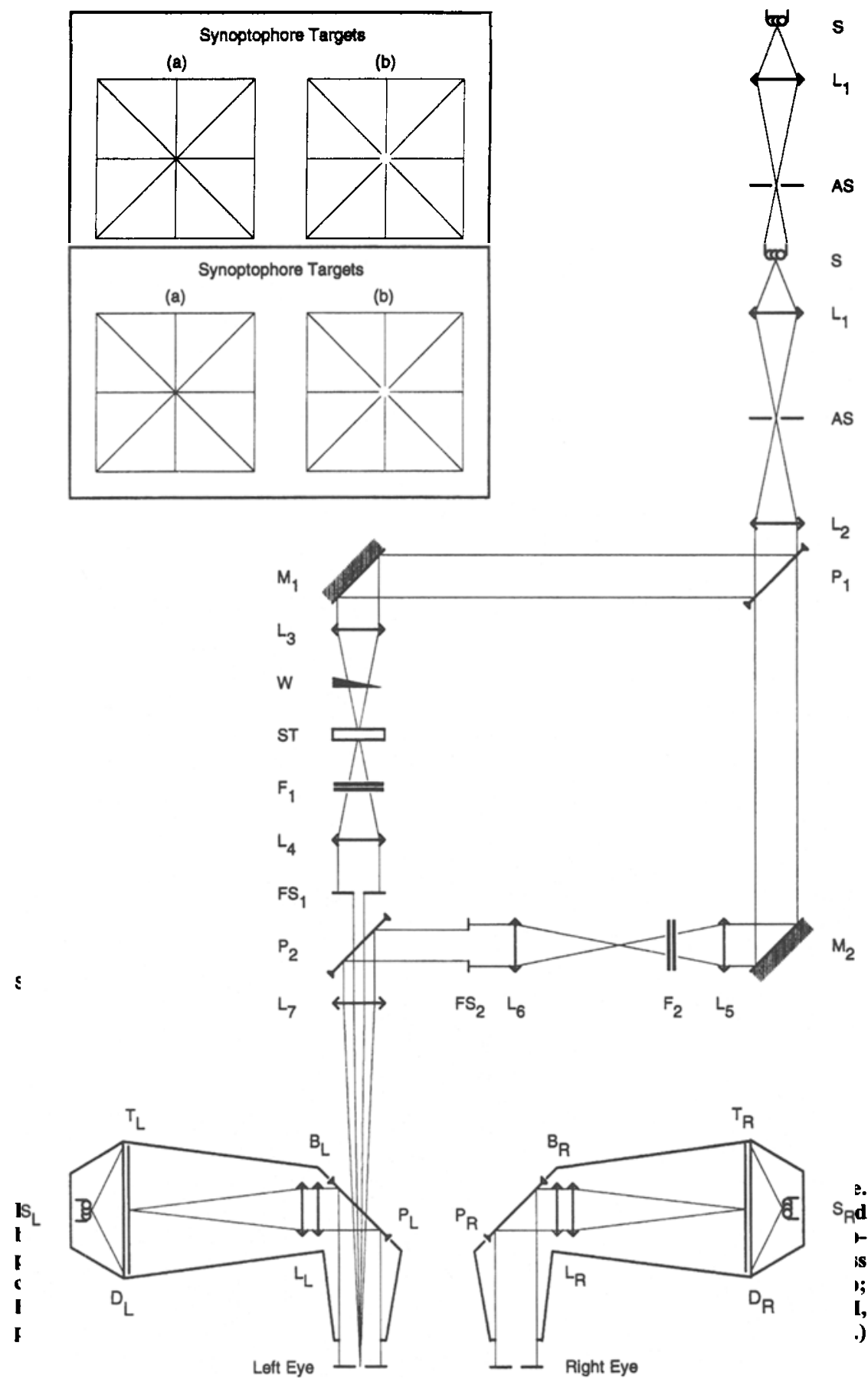

are used, in an attempt to tap into the sustained and transient channels: The transient mechanism should be more sensitive to brief-duration stimuli, and the sustained mechanism should be more sensitive to long-duration stimuli. Measuring reaction times as a function of stimulus duration also allows us to examine temporal summation at both threshold and suprathreshold levels for the two conditions. Reaction times have also been used to assess binocular rivalry suppression and to determine whether suppression is present during normal binocular vision (O'Shea, 1987; O'Shea \& Crassini, 1981).

\section{METHOD}

\section{Apparatus}

Temporal summation was measured with a binocular four-channel optical system, shown schematically in Figure 1. Two channels were part of a Maxwellian view optical system; the others were the left and right channels of a synoptophore. 
Table 1

Observer Details

\begin{tabular}{|c|c|c|c|c|}
\hline Observer & Age & Eye & Refractive Error & Corrected Acuity \\
\hline M.K. & 35 & $\begin{array}{l}\mathrm{L} \\
\mathrm{R}\end{array}$ & $\begin{array}{l}+0.25 /-0.50 \times 160 \\
\text { plano/ }-0.25 \times 80\end{array}$ & $\begin{array}{l}6 / 4.5^{+} \\
6 / 4.5^{+}\end{array}$ \\
\hline M.P. & 25 & $\begin{array}{l}\mathbf{L} \\
\mathbf{R}\end{array}$ & $\begin{array}{l}+3.50 /-1.75 \times 160 \\
+3.75 /-1.75 \times 10\end{array}$ & $\begin{array}{l}6 / 6^{+} \\
6 / 6^{+}\end{array}$ \\
\hline G.N. & 25 & $\begin{array}{l}\mathrm{L}^{*} \\
\mathrm{R}\end{array}$ & $\begin{array}{l}+5.50 /-1.50 \times 70 \\
+0.75\end{array}$ & $\begin{array}{l}6 / 6^{-} \\
6 / 4.5\end{array}$ \\
\hline S.D. & 26 & $\begin{array}{l}\mathrm{L}^{*} \\
\mathrm{R}\end{array}$ & $\begin{array}{l}+5.00 /-4.50 \times 29 \\
+1.25\end{array}$ & $\begin{array}{l}6 / 19 \\
6 / 4.5\end{array}$ \\
\hline L.V. & 22 & $\begin{array}{l}\mathrm{L} \\
\mathrm{R}^{*}\end{array}$ & $\begin{array}{l}+0.50 /-0.25 \times 170 \\
+4.25 /-1.00 \times 180\end{array}$ & $\begin{array}{l}6 / 6^{+} \\
6 / 7.5^{+}\end{array}$ \\
\hline P.D. & 29 & $\begin{array}{l}\mathrm{L}^{*} \\
\mathrm{R}\end{array}$ & $\begin{array}{l}+1.25 /-3.50 \times 15 \\
+0.25 /-0.50 \times 170\end{array}$ & $\begin{array}{l}6 / 7.5^{--} \\
6 / 3.8\end{array}$ \\
\hline
\end{tabular}

*Amblyopic eye.

One channel of the Maxwellian view was used for the test field; the other channel provided a background field during pilot experiments in which the synoptophore background targets were not used. Anisometropia was induced by inserting positive lenses in the synoptophore at positions $B_{L}$ or $B_{R}$, out of the optical path of the test field. The combination of the test field and the synoptophore targets was made possible by the replacement of the plane mirrors in the synoptophore with pellicles $P_{L}$ and $P_{R}$. Presentation of the test field to the left or right eye was achieved by sliding the synoptophore along rails to the right or left, respectively. Normal synoptophore controls for interpupillary distance and phorias were available and permitted precise alignment of the targets.

Experiments were automated via a personal computer (IBM compatible) fitted with a digital input/output board (PC-14B; Boston Technology) and a sound cara (SoundBlaster Pro, Version 2.0, Creative). The experimental paradigm was controlled by a $\mathrm{C}++$ program (Borland $\mathrm{C}++$, Version 3.1). The digital input/output board was used to operate the shutter (Uniblitz), monitor the observer's responses, and accurately time the stimulus and foreperiod durations (using an on-board timer). The sound card generated ready signals, feedback signals, and white noise in headphones worn by the observer. The observer's head was held steady by having the observer bite on a dental wax impression (Kerr) connected by a rigid bar to the synoptophore.

\section{Stimuli}

Background field targets are shown in Figure 1 (inset). The line width for each target was $0.55^{\circ}$, and the missing central region of target (b) was $1.00^{\circ}$ in diameter. Under monoptic conditions, only target a was viewed; the other eye was occluded. Under dichoptic conditions, both targets were used: target a was viewed by the eye being tested, the other eye viewed target $b$. Both background fields had the same luminance and gave a retinal illuminance of 1,720 td (based on a dilated pupil size of $8 \mathrm{~mm}$ for each observer). This value ensured that observers were at least $2 \mathrm{log}$ units into the region of Weber adaptation (Blackwell, 1946; Fuortes, Gunkel, \& Rushton, 1961).

The test field was a $0.65^{\circ}$ diameter disk. Foveal spatial summation (i.e., critical area) reported for amblyopic eyes is smaller than this value (Flynn, 1967; Grosvenor, 1957; Miller, 1955; Weber, 1988); thus, any changes in spatial summation should not affect thresholds for observers with anisometropic amblyopia. The test was presented monocularly in the center of the continuous background target as a rectangular pulse. Illuminance was measured after each session with an illuminance meter (Gossen PANLUX), and retinal illuminance was calculated with the method described by Nygaard and Frumkes (1982). The test field was attenuated by fixed neutral density filters and a circular neutral density wedge.

Stimulus duration was calibrated with a digital storage CRO (Gould $20 \mathrm{MHz}$, Type 1425) and a photodiode (Radiometric filter, Model 1158, United Detector Technologies). At onset, the lumi- nance rose to maximum in $3 \mathrm{msec}$; at offset, the luminance fell to zero in $1.5 \mathrm{msec}$. All flash durations reported were calculated by the width-at-half-height of the pulse.

\section{Observers}

Six observers participated in this experiment; each gave informed consent. Two of these, the authors (M.K. and M.P.), had normal (corrected) visual acuity and normal binocular vision; both had knowledge of the purpose of the experiments. Four observers had hypermetropic anisometropic amblyopia. Two of the amblyopic observers (G.N. and S.D.) had a general idea of the purpose of the experiments; the other two (L.V. and P.D.) were naive.

The diagnosis of anisometropic amblyopia was based on our clinical assessment, interpreted in terms of the following definition proposed by Ciuffreda, Levi, and Selenow (1991):

\begin{abstract}
Amblyopia can be defined as a unilateral (or infrequently bilateral) condition in which the best corrected visual acuity is less than $6 / 6$ in the absence of any obvious structural or pathological anomalies but with one or more of the following occurring before the age of 6 years: amblyogenic anisometropia, constant unilateral esotropia or exotropia, amblyogenic bilateral isometropia, amblyogenic unilateral or bilateral astig. matism, image degradation.
\end{abstract}

Each observer was orthophoric at the time of testing. One of the amblyopic observers (G.N.) experienced intermittent exotropia during childhood and when fatigued but usually showed no eye movement to cover test. None of the other observers had a history of strabismus or showed any abnormality of ocular alignment on examination. None of the observers had anomalous retinal correspondence (as tested by the Bagolini striated glasses). The presence of reduced corrected monocular vision in the absence of any other ocular abnormality justifies the inference that anisometropia was present early in life.

Both of the observer's pupils were dilated (two drops of $0.5 \%$ Tropicamide) $15 \mathrm{~min}$ prior to the experiment, and full spectacle corrections were worn. Visual angles were not corrected for magnification effects. Details for each observer are supplied in Table 1.

\section{Procedure}

Prior to formal data collection, each observer was provided with two or more sessions of practice, in order to minimize learning effects during the course of the experiment. Also, prior to each session, a short practice $(5-10 \mathrm{~min})$ was conducted to ensure proper alignment of the observers and to prepare them for the main session. Observers wore earplugs and headphones playing white noise to mask the sound of the shutter and other extraneous noises. The ready signals and warning signals were also supplied through the headphones. An experimental session consisted of 10 runs for up to seven stimulus durations and lasted from 1.5 to $2.5 \mathrm{~h}$.

Increment thresholds were determined as a function of stimulus duration with a modified method of limits that incorporated a simple reaction time paradigm (Harwerth et al., 1980; Kalloniatis \& Harwerth, 1990, 1991). In each trial, after a ready signal (a brief tone), the observer depressed a hand-held response button that initiated an exponentially distributed foreperiod with a mean wait of $500 \mathrm{msec}$. (This type of foreperiod has a constant hazard function, which means that the time elapsed since the warning signal does not affect the momentary tendency for the reaction stimulus to appear; thus, there are no cues for the observer as to when to expect the reaction stimulus.) The stimuli were presented for a range of durations that varied pseudorandomly. Each stimulus offset occurred either after the duration of the stimulus elapsed or after the response of the observer (i.e., it was response-terminated), whichever occurred first. Therefore, the stimulus duration specified in the experiments was the maximum duration. (Note that, except for the longest duration stimulus at intensities far above threshold, the maximum duration always occurred.) Observers were instructed to respond as quickly as possible to the stimulus, without making early releases. If the observer released the button within the response interval (starting $100 \mathrm{msec}$ 


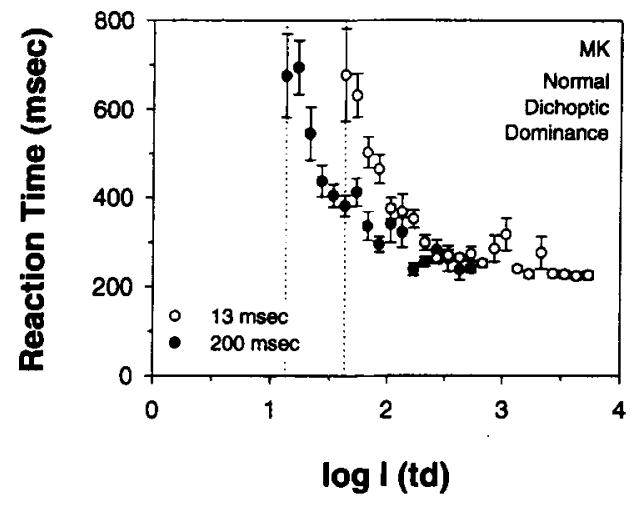

Figure 2. Mean reaction time (in milliseconds) plotted as a function of intensity for two durations, measured under the conditions indicated (Observer M.K.). Error bars show standard errors of the mean. Reaction times are shortest for the highest test intensity and then increase as test intensity is decreased toward threshold (indicated by the vertical dotted lines). Thresholds for the 13- and 200-msec test flashes occur at 1.63 and $1.13 \mathrm{log}$ td, respectively. Subsequent reaction time figures have been normalized (by translating the data along the $x$-axis to position threshold at $1.0 \log$ td) to simplify comparison of the shape of the functions.

after stimulus onset and lasting $1,000 \mathrm{msec}$ after stimulus offset), it was assumed that the observer detected the stimulus (a hit), and the reaction time was recorded as the time from the onset of the stimulus to the observer's button release. The response was reinforced with a brief high-pitched tone. A response during the foreperiod or within $100 \mathrm{msec}$ of the stimulus onset (an early release) was penalized by a long-duration deep tone (time-out of $5 \mathrm{sec}$ ). If there was no response before the end of the response interval (a miss), it was indicated by a brief deep tone. After feedback, there was a 1-sec delay before the ready signal of the next trial. After each trial that resulted in a hit, the intensity of the stimulus was decreased by $0.1 \log$ units. For each consecutive miss after the first miss after a hit, there was a .5 probability that the run would terminate. Alignment of the observer was checked after each run.

As noted in the introduction, induced anisometropic suppression may not result in constant suppression of the more defocused eye. For the normal observers, near to constant suppression was achieved with $2.0,3.0$, and 6.0 dioptres of anisometropia. In order to produce constant suppression in the nonamblyopic eye of the observers with anisometropic amblyopia, we were forced to use 6.0 dioptres of anisometropia. We used the same amount of anisometropia for the amblyopic eye. Even with these levels, there were rare occasions on which suppression and dominance would alternate. To maximize the chance that stimuli were presented during the phase we were interested in, observers were advised to initiate a trial at the beginning of the suppression or dominance phases. Distributions for the durations of the rivalry-like phases were measured subjectively with a timer (Fox, Todd, \& Bettinger, 1975; Levelt, 1965; Walker, 1975). These distributions were fitted with gamma distribution (Lehky, 1988) and compared with the foreperiod distribution. In all cases (except for Observer M.K. with 1.0 dioptre of anisometropia, see Results), there was less than $6 \%$ overlap (i.e., on average, the stimulus may have been presented during the wrong phase in only 1 out of 17 trials).

There were three situations examined in this experiment: monocular, dominance, and suppression. No suppression can occur in the monocular condition (by definition, suppression is a purely binocular phenomenon), so it is used as a control condition for 2 of the observers with amblyopia. The dominance condition is achieved when both eyes view the background targets, with the anisometropia blurring the background for the eye not being tested, forcing the tested eye to be dominant. The suppression condition is the opposite, with the background blurred for the eye being tested, forcing it to suppress.

\section{Analysis}

For each condition, mean reaction times were plotted as a function of intensity. Piéron $(1914,1920)$ was first to suggest that these functions follow a very simple law:

$$
E(I)=r_{0}+k I^{-\beta},
$$

where $E(I)$ is the expected reaction time, $I$ is a measure of intensity, $r_{0}$ is an irreducible time constant, $k$ is a reducible time constant, and $\beta$ is an exponent. Mansfield (1973) and Kohfeld, Santee, and Wallace (1981a, 1981b) have presented various methods for fitting this empirical formula to the data. However, more recently, Luce (1986) has proposed that a better method is to fit Equation 1 to the data with a least squares fitting procedure. Luce compared results of the three methods and found that, depending on the method used, the value of $r_{0}$ could be affected by tens of milliseconds and, more importantly, $\beta$ by factors of 2 or 3 . We fitted our reaction time data with the least squares method suggested by Luce.

For each nominal duration, psychometric functions were derived from the number of responses obtained at each stimulus intensity during the method of limits. A cumulative Gaussian with two freefloating parameters (mean and standard deviation) was fitted to these psychometric functions (SigmaPlot for Windows, Jandel Scientific). Threshold was defined as the intensity required to produce $50 \%$ correct (i.e., the mean of the fitted Gaussian) and has been plotted against duration in Figure 6 and in the first column of Figure 7. Since analysis of threshold-duration functions does not provide a useful description of the temporal response of a linear system (Watson, 1986), we used a classical two-line curve fit with two free parameters: the critical duration $\left(t_{\mathrm{c}}\right)$ and the critical intensity $\left(I_{\mathrm{c}}\right)$. Equation 2A models the Bloch's law (or total temporal summation) region of the curve and has a fixed slope of -1 . Equation $2 \mathrm{~B}$ models the region of zero temporal summation and has a fixed slope of zero.

$$
\begin{array}{ll}
\log I=\log I_{\mathrm{c}}+\log t_{\mathrm{c}}-\log \mathrm{t} & \text { for } t \leq t_{\mathrm{c}} \\
\log I=\log I_{\mathrm{c}} & \text { for } t>t_{\mathrm{c}}
\end{array}
$$

In these equations, $I$ is the threshold intensity and $t$ is the duration.

We also fitted threshold-duration functions with the following function:

$$
I / I_{\mathrm{c}}=1+t_{\mathrm{c}} / t
$$

A useful property of this function is that it has a smooth transition between the regions of complete and zero temporal summation. However, the goodness-of-fit (as assessed by chi-squared) was not consistently better with Equation 3 than with Equations $2 \mathrm{~A}$ and $2 \mathrm{~B}$. For this reason and to enable comparison with previously published data, we have used Equations $2 \mathrm{~A}$ and $2 \mathrm{~B}$ to model our thresholdduration data.

\section{RESULTS}

\section{Reaction Times}

Figure 2 shows the reaction times as a function of test intensity for 13- and 200-msec test durations (measured under the conditions indicated). Reaction time versus intensity functions have the same basic properties for all conditions: The reaction time is shortest for the highest test intensity and then increases as test intensity is decreased toward threshold (indicated by the vertical dotted lines). The 13-msec data appear to the right of the 200-msec data, because the threshold for the $13-\mathrm{msec}$ test flash is $0.5 \mathrm{log}$ units higher than the threshold for the 200-msec test flash (compare to 


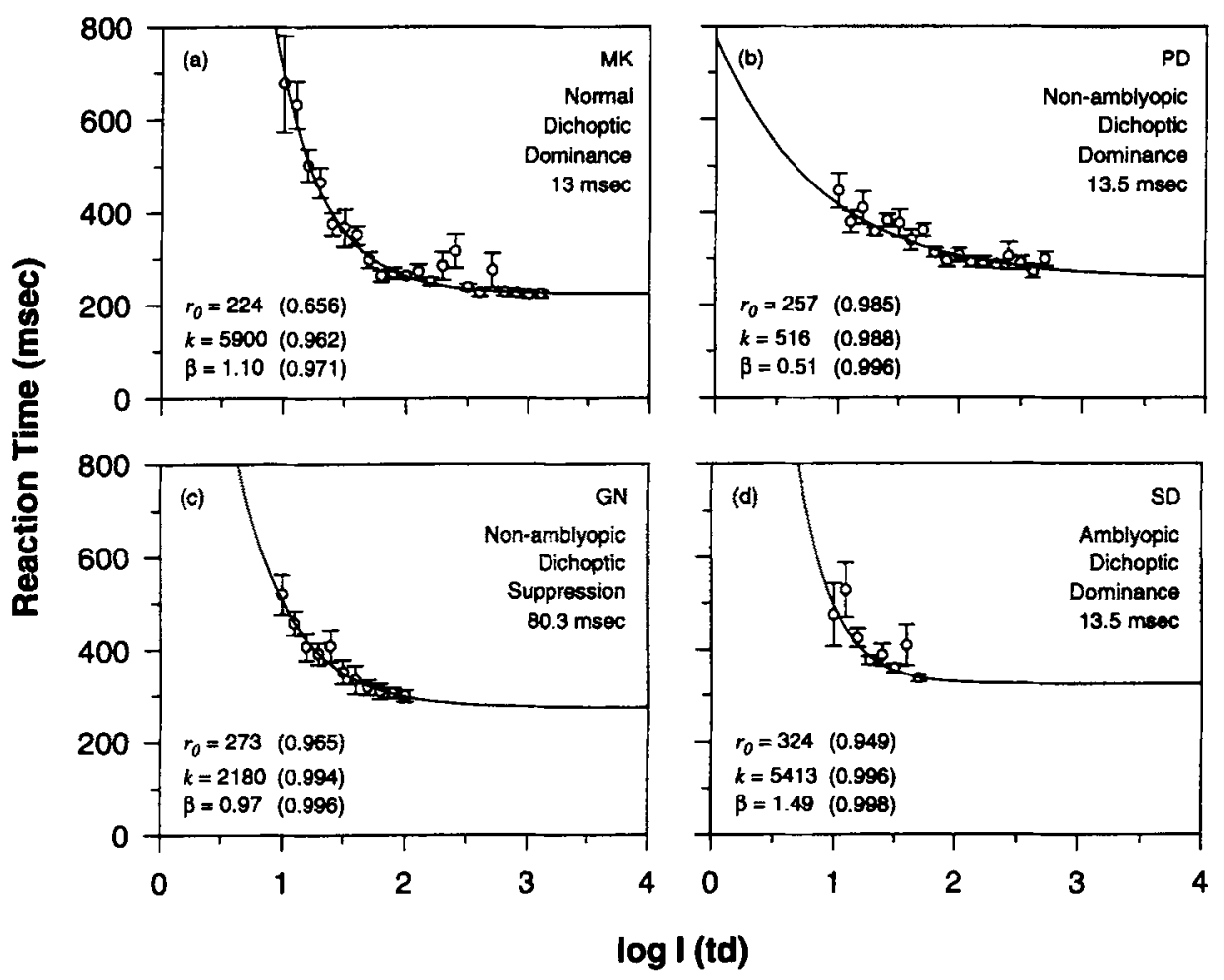

\begin{abstract}
Figure 3. Mean reaction time (in milliseconds) plotted as a function of intensity for various observers and conditions. Error bars show standard errors of the mean. The lines represent the results of the curve fit using the method suggested by Luce (1986), and the parameters and their dependencies (in parentheses) are shown. The parameters $k$ and $\beta$ (and often $r_{0}$ ) have dependencies close to one, indicating that the equation is over-parameterized.
\end{abstract}

Figure $6 \mathrm{a}$, open circles). Figures $3 \mathrm{a}-3 \mathrm{~d}$ and $4 \mathrm{a}-4 \mathrm{~d}$ show similar data, but these data have been normalized by translation along the $x$-axis to position threshold test intensity at $1.0 \log$ td in order to simplify comparison of the function shapes (the data shown in Figure 4a are the normalized data of Figure 2). At near threshold test intensities, typical reaction times were between 500 and $800 \mathrm{msec}$.

To obtain parameters that could be used to characterize and compare the shape of the mean reaction time versus intensity functions, we fitted the data with the equation suggested by Luce (1986). However, the two shape parameters $(k$ and $\beta$ ) derived with this procedure were not independent. Figure 3 illustrates this dependency problem when applied to select data from a variety of conditions and observers. The values of the parameters returned from the curve fit are indicated on each graph; the dependencies are shown in parentheses. The dependence of a parameter is defined to be

dependence

$=\frac{\text { (variance of the parameter, other parameters constant) }}{\text { (variance of the parameter, other parameters changing) }}$.

The parameters $k$ and $\beta$ (and often $r_{0}$ ) have dependencies near one, suggesting that they are strongly dependent on one another. This indicates that the equation is too complicated and is probably over-parameterized. Note that higher dependencies result when the data are variable or when the data are collected over a smaller range of intensities. However, even with data obtained from an experienced observer over a wide range of intensities, $k$ and $\beta$ are still dependent on each other (Figure 3a). Thus, $k$ and $\beta$ alone should not be used to describe the shape of these functions.

As the approach proposed by Luce (1986) could not be used to uniquely describe the shape of the functions and as previously used methods have also been shown to be inadequate, we developed a new method for comparing mean reaction time versus intensity curves. Graphs comparing two parameters were plotted (nonamblyopic vs. amblyopic, monocular vs. dominant, dominant vs. suppressed, or durations inside Bloch's law vs. durations outside Bloch's law), and separate graphs were produced for each combination of the other parameters. The threshold values of both functions were normalized to $1 \log$ td. In other words, the reaction time versus intensity curves being compared were translated along the $x$-axis, so that threshold corresponded to $1 \log$ td. This superimposed the functions for the two conditions and exposed any shape differences.

To further emphasize shape differences, difference plots (or visuograms) were derived. These graphs plot the difference in mean reaction time as a function of intensity for each of the graphs discussed above. If the mean reac- 

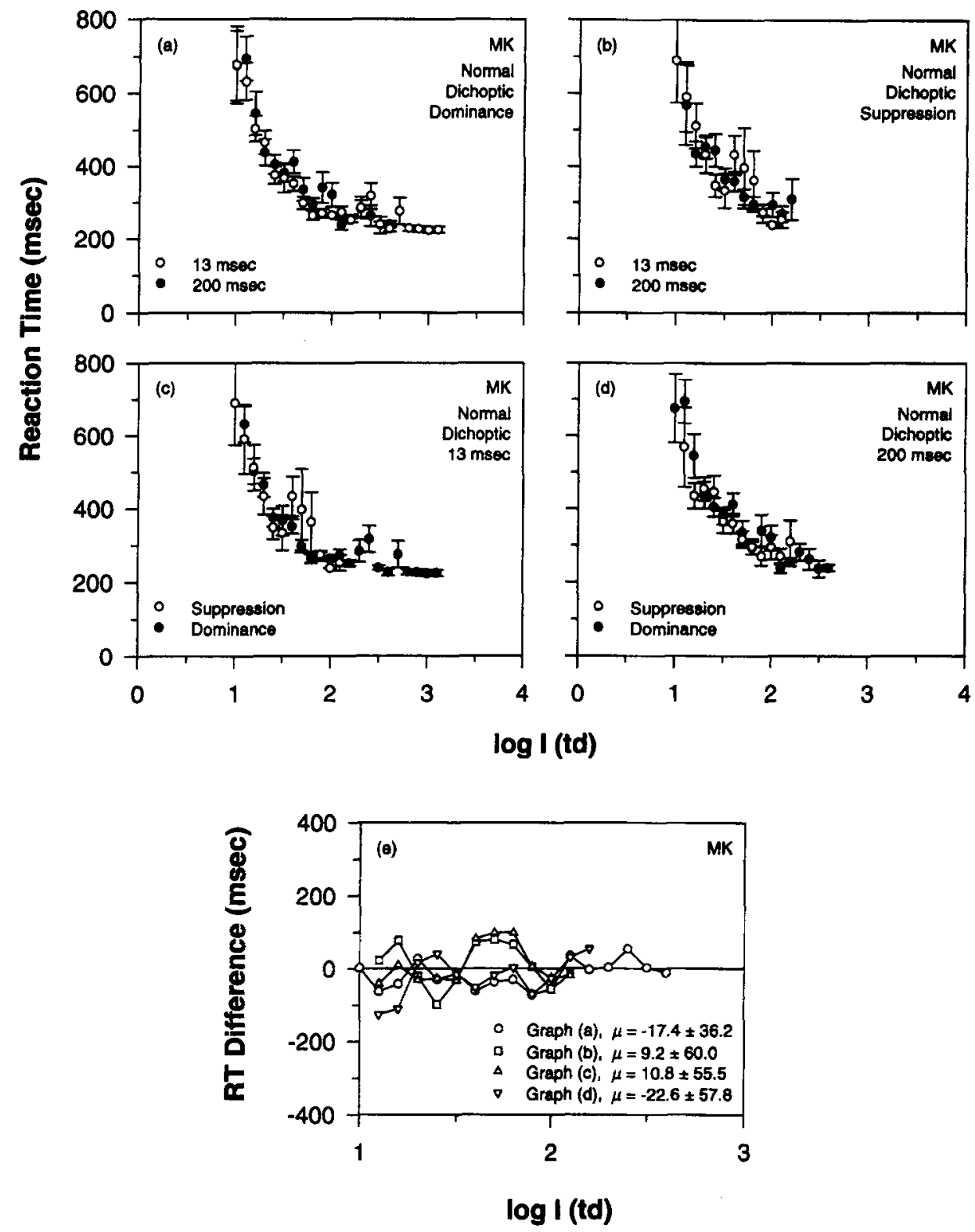

Figure 4. Mean reaction time (in milliseconds) plotted as a function of intensity (normalized relative to $1 \mathrm{log}$ td) for normal Observer M.K. Error bars show standard errors of the mean. Panels ab: A comparison of durations within and outside Bloch's law, during dominance and suppression. Panels c-d: A comparison of suppression and dominance for durations within and outside Bloch's law. Panel e: A visuogram showing the difference in mean reaction time between the functions plotted on each of the other graphs (open symbols minus filled symbols). The visuogram shows no systematic variation, indicating that the mean reaction time versus intensity curves are the same shape for each condition.

tion time versus intensity functions are different shapes, there will be a systematic variation in the visuogram plot. If there is a constant increase or decrease of the mean reaction time for one of the functions, there will be a shift up or down of all points on the visuogram. To highlight these types of changes, the mean and standard deviation for each plot as a whole are indicated in the figure legend.

Figure 4 shows mean reaction time plotted as a function of normalized intensity for Observer M.K. Figures $4 a-4 d$ show that there is no difference in the shapes of the functions for the two conditions, and this is confirmed by the visuogram (Figure 4e). The similarity of the shapes of the functions indicates that it is likely that detection is mediated by the same mechanism, under these experimental conditions (Harwerth \& Levi, 1978a, 1978b).

Figure 5 shows visuograms for the amblyopic Observers G.N., S.D., L.V., and P.D., respectively. Only a selection of parameter combinations has been shown for each of these 


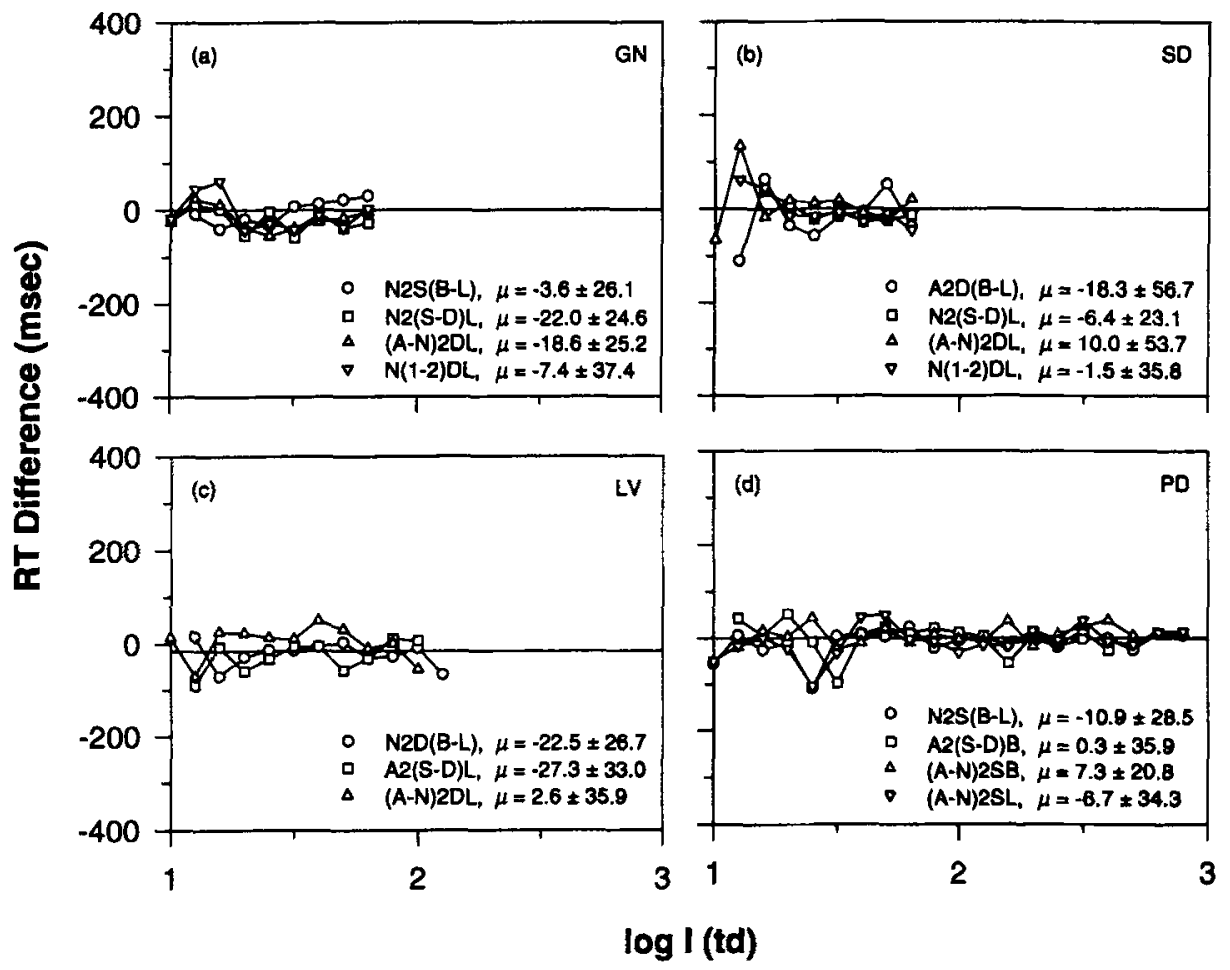

Figure 5. Visuograms for amblyopic Observers G.N., S.D., L.V., and P.D. The legend contains a key and the mean and standard deviation for each plot separately. The visuograms show no systematic variation, indicating that the mean reaction time versus intensity curves are the same shape for each cordition. [Legend key: Each legend entry contains five characters. The two parameters that are compared in the visuogram are shown in parentheses. Therefore, the key can be considered in four sections, in the following order. Eye tested: $N$, nonamblyopic; $A$, amblyopic. Viewing condition: 1, monoptic; 2, dichoptic. Suppression phase: $S$, suppression; D, dominance. Stimulus duration: B, brief (13.5 msec); L, long (80.3 msec). The two parameters that are compared are shown in parentheses.]

observers, because the results are the same for all conditions. The shapes of the functions are very similar for every combination of parameters in each observer. Thus, in both amblyopic and nonamblyopic eyes, it is likely that detection is mediated by the same mechanism (Harwerth \& Levi, 1978a, 1978b).

There is some suggestion that longer duration stimuli result in longer reaction times, because the mean of the mean reaction time differences for these conditions are all negative; however, this effect is small. For the dominant and the suppressed condition, the mean reaction times of the normalized curves are the same. Thus, the mean of the reaction time differences for these conditions are close to zero. Therefore, when the intensity (or Weber contrast) is normalized to equate threshold, there is no difference in reaction time for the dominant condition and the suppressed condition. The same applies for the amblyopic and nonamblyopic eyes.

\section{Temporal Summation}

Threshold-duration functions were plotted with thresholds derived by the procedure outlined in the Method section. Similar functions, but for suprathreshold stimuli, were derived from the reaction time data by plotting the test intensity for a criterion reaction time at each duration. The shape-invariant nature of the reaction time versus intensity functions predicts that the suprathreshold results would have the same critical duration $\left(t_{\mathrm{c}}\right)$ as the threshold results, but with critical intensity $\left(I_{c}\right)$ increasing as criterion reaction time decreased. Plots of supratheresholdduration functions (not shown) confirmed this prediction. Consequently, we discuss only the threshold data below.

Figure 6 shows dichoptic threshold-duration functions for 2 normal observers under various levels of induced anisometropia (0-3.0 dioptres). The dominance condition (no anisometropia) is an example of a normal thresholdduration function. The critical durations are within the normal range expected at this level of adaptation, as are the critical intensities (Roufs, 1972). For the suppressed condition ( 3.0 dioptres of anisometropia), there is a general increase in threshold at all durations (by about $0.6 \mathrm{log}$ units), as compared with the control condition. In other words, $I_{\mathrm{c}}$ increases while $t_{\mathrm{c}}$ remains the same.

For Observer M.K., thresholds measured for $2.0,3.0$, and 6.0 (not shown) dioptres of anisometropia were all similar. The same result was found for Observer M.P. with 3.0 


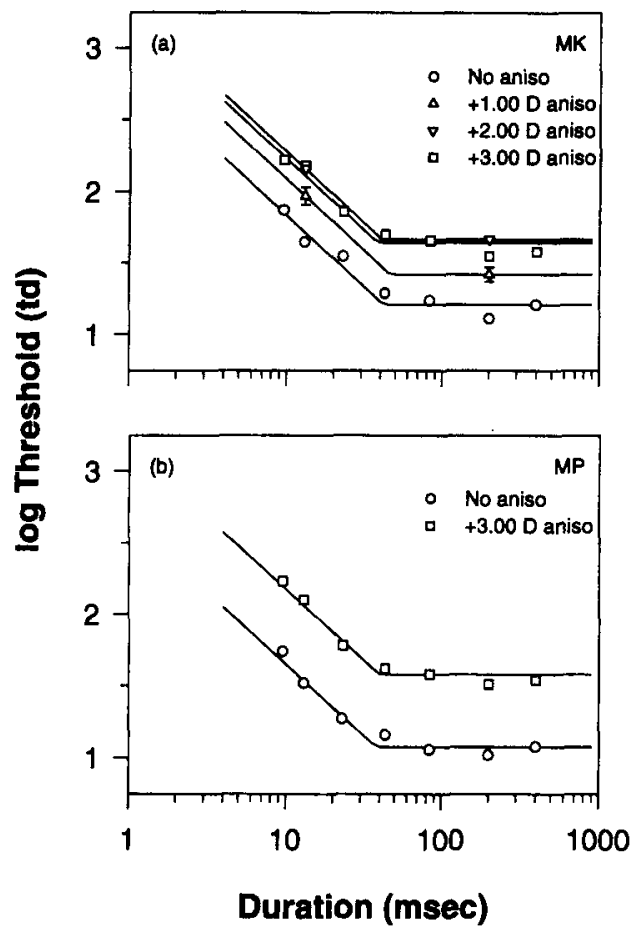

Figure 6. Threshold-duration functions for two normal observers under dichoptic conditions for various levels of anisometropia (indicated in the legend). Standard errors of the mean are smaller than the symbol size, except where indicated. Solid lines through the data represent the curve fitting results. Induced anisometropic suppression causes an equal increase in threshold at all durations. The results for 6.0 dioptres of anisometropia were similar to the results for 3.0 dioptres of anisometropia and are not shown, for clarity.

and 6.0 (not shown) dioptres of anisometropia. This suggests that anisometropic suppression is complete with 2.0 dioptres of anisometropia and that further increases in the level of anisometropia will not result in a change to the suppression. The results for 1.0 dioptre of anisometropia lie between the dominant and suppressed conditions. For this condition, the observer reported frequent alternations of suppression and dominance, similar to binocular rivalry. Standard errors of the mean for these points are larger than those for the other results, which is to be expected because, for this condition, the test flash is sometimes presented during suppression and at other times during dominance. This will result in the mean threshold lying somewhere between the mean threshold for dominance and suppression (in a position dependent on the temporal characteristics of the alternations).

Threshold-duration functions for the 4 amblyopic observers (G.N., S.D., L.V., and P.D.) are shown in the first column of Figure 7. All observers display similar characteristics. Results for the nonamblyopic eye show that the threshold-duration function for the monocular condition (Observers G.N. and S.D. only) is very similar to that for the dominance condition (both $I_{\mathrm{c}}$ and $t_{\mathrm{c}}$ are similar). However, with suppression, there is a general increase in threshold (by $0.4-0.6 \log$ units), with a minimal increase in $t_{\mathrm{c}}$. These results compare favorably with the results for normal observers. The results for the amblyopic eye indicate that the thresholds for the monocular condition (Observers G.N. and S.D. only) are again similar to those obtained for the dominance condition. However, with suppression, there is a general increase in threshold (by $0.1-0.4 \log$ units) and again a minimal increase in $t_{\mathrm{c}}$. There is little difference in $t_{\mathrm{c}}$ between nonamblyopic and amblyopic eyes under any of the conditions. That is, for each condition there is a general difference in threshold for the amblyopic eye, as compared with the nonamblyopic eye. For the monocular condition, this difference is $0.5-0.6 \log$ units; for the dominant condition, the difference is $0.3-0.6 \log$ units; and for suppression, the difference is only $0.1-0.2 \log$ units.

The second column in Figure 7 illustrates the changes that occur in the critical intensity. There is only a small change (less than $0.2 \log$ units) in $I_{\mathrm{c}}$ for the monocular and dominance conditions. However, suppression causes a common pattern of change: $I_{c}$ increases for both the nonamblyopic and amblyopic eyes, with this increase being greater for the nonamblyopic eye. Therefore, the threshold elevation that accompanies suppression results in the threshold-duration functions becoming similar for the nonamblyopic and amblyopic eyes. To facilitate interpretation of these results in terms of the Weber contrast, Table 2 shows the converted $I_{\mathrm{c}}$ values.

The last column of Figure 7 highlights the constancy of the critical duration. There is no difference between $t_{c}$ for the monocular condition and $t_{\mathfrak{c}}$ for the dichoptic condition, and suppression does not cause any reliable pattern of change in $t_{\mathrm{c}}$. Some observers show an increase in $t_{\mathrm{c}}$ with suppression; others show a decrease; however, these changes are small (not more than $0.2 \mathrm{log}$ units). The nonamblyopic eyes tend to have a longer $t_{c}$ than do the amblyopic eyes; however, these differences are also small (not more than $0.2 \log$ units). Therefore, temporal integration does not appear to be notably affected by either induced anisometropic suppression or anisometropic amblyopia.

\section{DISCUSSION}

This study presents reaction time and temporal summation data for 4 observers with anisometropic ambly-

Table 2

The Critical Intensity $\left(I_{\mathrm{c}}\right)$ Expressed in Terms of Weber Contrast

\begin{tabular}{clccc}
\hline & & \multicolumn{3}{c}{ Weber Contrast $(\Delta I / I)$} \\
\cline { 3 - 5 } Observer & \multicolumn{1}{c}{ Eye } & Monoptic & Dominance & Suppression \\
\hline \multirow{2}{*}{ G.N. } & Nonamblyopic & 0.4 & 0.5 & 2.0 \\
& Amblyopic & 1.5 & 1.0 & 3.2 \\
S.D. & Nonamblyopic & 0.5 & 0.5 & 1.9 \\
& Amblyopic & 1.8 & 2.1 & 3.0 \\
L.V. & Nonamblyopic & & 0.7 & 1.8 \\
& Amblyopic & & 2.3 & 2.7 \\
P.D. & Nonamblyopic & & 0.4 & 2.0 \\
& Amblyopic & & 1.1 & 2.3 \\
\hline
\end{tabular}

Note-All values are percentages. 
Threshold-duration

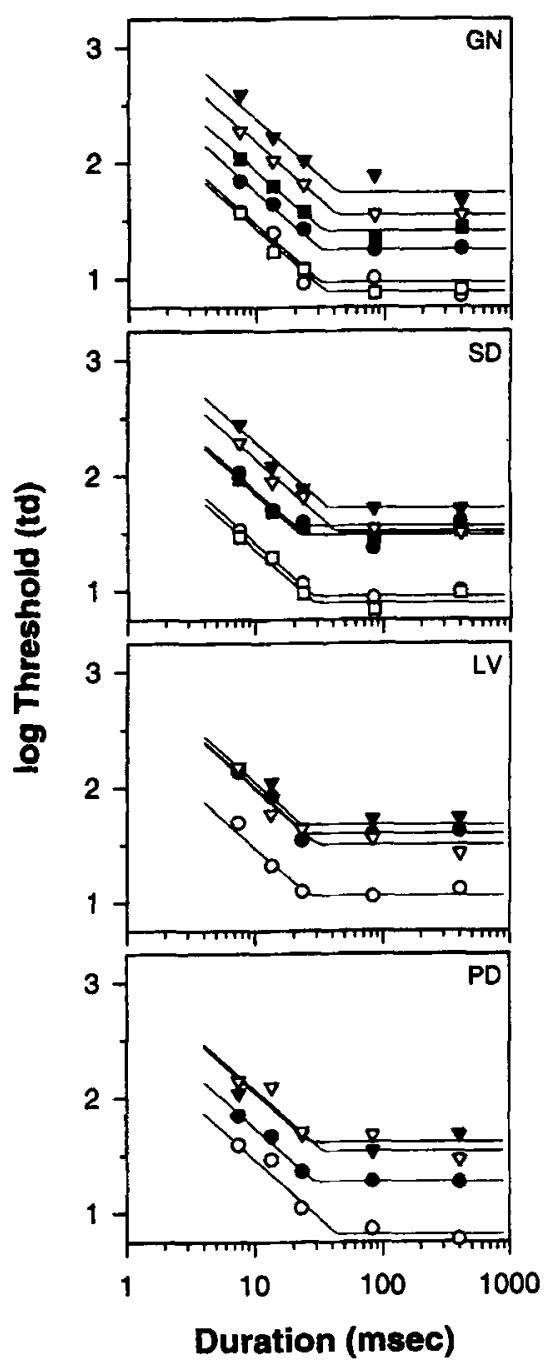

\section{Critical Intensity}
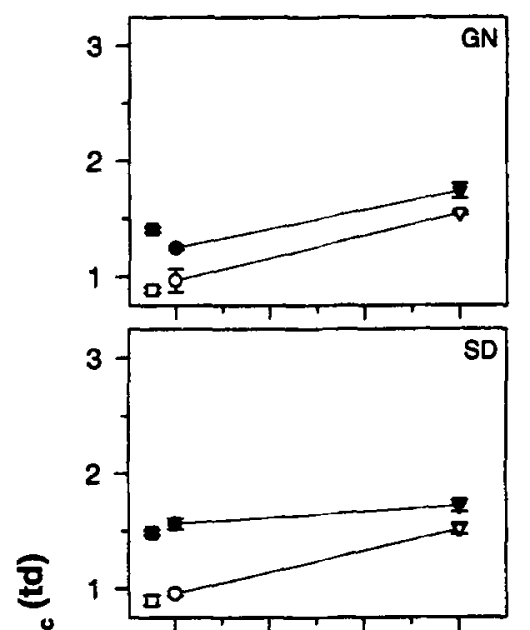

뭉
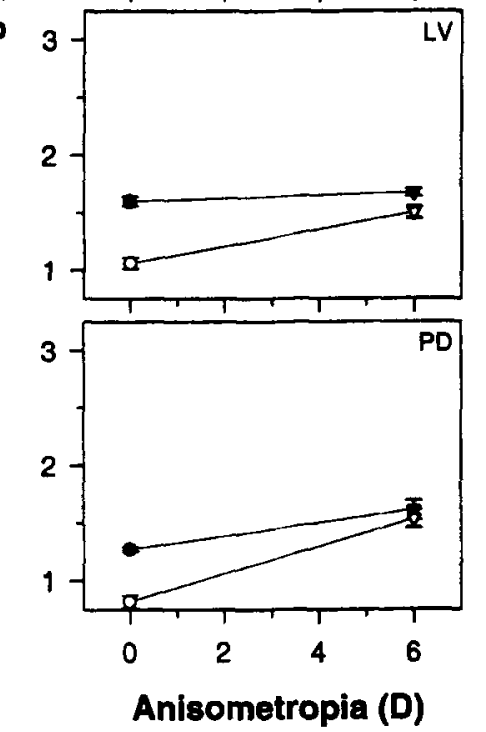

Critical duration

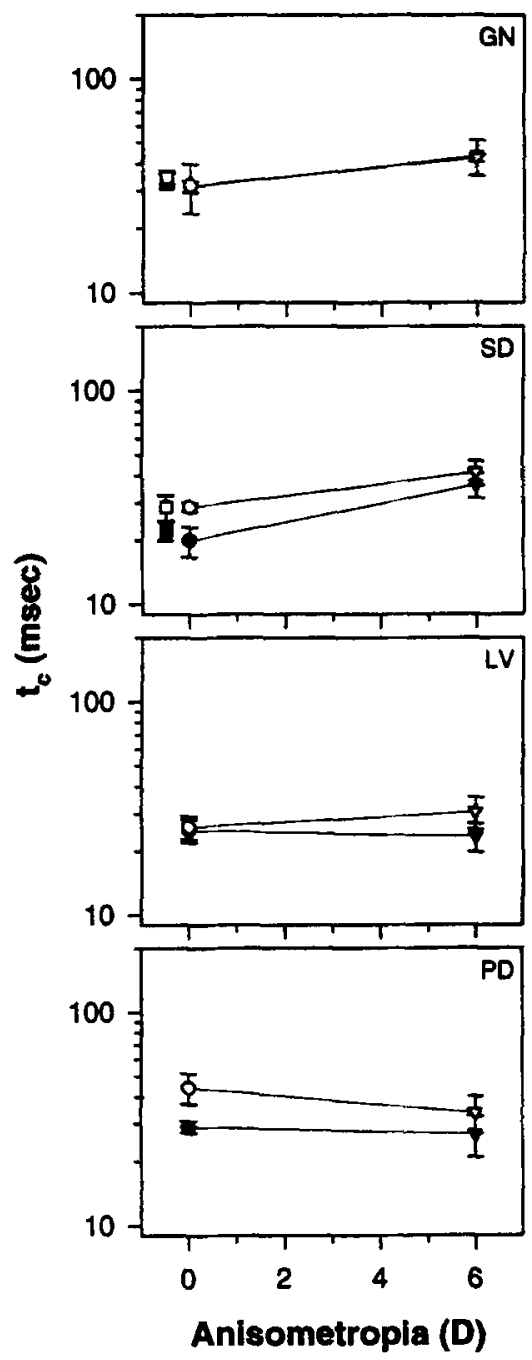

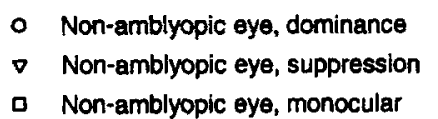

- Amblyopic eye, dominance

- Amblyopic eye, suppression

- Amblyopic eye, monocular

Figure 7. Threshold-duration functions, critical intensities $\left(I_{c}\right)$, and critical durations $\left(t_{c}\right)$ for 4 observers with anisometropic amblyopia. The eye tested and the viewing condition are identified in the legend. Amblyopia and induced anisometropic suppression both cause an equal increase in threshold at all durations. There is no notable change in the critical duration for any condition. With induced anisometropic suppression the critical intensity increases more for the nonamblyopic eye than it does for the amblyopic eye.

opia, on the basis of the criteria of Ciuffreda et al. (1991) and our clinical assessment. However, we acknowledge that the use of a traditional clinical definition of amblyopia (e.g., a two-line difference in acuity) would not classify 2 of the observers (L.V. and G.N.) as amblyopic. Given the definition noted above and other data (see Results), we consider these to be cases of mild amblyopia. We regard Observer P.D. to be a case of moderate amblyopia and Observer S.D. to be a case of more severe amblyopia. Thus, we have attempted to cover a range of amblyopia severity.

\section{Reaction Times}

The data obtained indicate that mean reaction time versus intensity functions obey the principles of shape invariance (i.e., only the curves' relative position along the $x$-axis changes, not the shape of the curve) for a variety of conditions: duration within Bloch's law versus duration outside Bloch's law, dichoptic (dominance) versus monocular, dominance versus suppression, and nonamblyopic versus amblyopic eye. The principle of shape invariance means that, for all criterion reaction times, the horizontal 
displacement between the curves will be the same, indicating the same relative effect at suprathreshold levels.

For any of the conditions of this study, there does not appear to be a difference in shape between the curves for a duration inside Bloch's law and those for a duration outside Bloch's law. Therefore, there is no change to temporal summation $\left(t_{\mathrm{c}}\right)$ at suprathreshold levels (if using a criterion reaction time paradigm) for any of these conditions.

These results are in conflict with those of Ueno (1977, 1978), who demonstrated that the exponent of these functions decreased with increasing stimulus duration. He found a monotonic increase in $t_{\mathrm{c}}$ with increasing criterion reaction time and different exponents for different conditions. There are several possible reasons for the difference between our data and the results of Ueno. First, Ueno (1977) used a constant foreperiod design, which results in a hazard function that increases with time. This type of foreperiod is unsatisfactory, because there is evidence for response criterion changes with a changing hazard function. In other words, this design allows the observer to base his or her response on an estimate of the foreperiod rather than on only the reaction stimulus (Luce, 1986). Catch trials are often used to suppress time estimations; however, Ueno (1977) did not use catch trials. Second, in both studies, Ueno $(1977,1978)$ did not give feedback to the observers. Thus, the observers do not have the opportunity to establish a value for the tradeoff between errors and speed (Luce, 1986); consequently, their criteria may vary. Finally, the fitting paradigm used by Ueno $(1977,1978)$ is unreliable (Luce, 1986).

There are differences in mean reaction time between the amblyopic and the nonamblyopic eyes before normalization, with the difference becoming smaller at higher intensities or contrasts. This finding is in agreement with numerous studies (Ciuffreda et al., 1991; Hamasaki \& Flynn, 1981; Mackenson, 1958; von Noorden, 1961). However, there appear to be no differences in mean reaction time between the amblyopic and the nonamblyopic eyes when the intensity is normalized to equate thresholds. It seems that the increase in reaction time for the amblyopic eye is a result of the contrast threshold deficit of that eye alone, and, if contrast is scaled in threshold units, the reaction times for the two eyes are equivalent.

\section{Temporal Summation}

Threshold-duration functions obtained for the nonamblyopic eye during monocular viewing are similar to those obtained for the normal and nonamblyopic eyes during dominance. For these conditions, critical durations $\left(t_{\mathrm{c}}\right)$ range from 20 to $42 \mathrm{msec}$, critical intensities $\left(I_{c}\right)$ range from 6.5 to $11.7 \mathrm{td}$, and Weber contrast ranges from $0.4 \%$ to $0.7 \%$. These parameters are within the ranges obtained by other authors for normal observers (under monocular conditions) at similar background intensities (Graham \& Kemp, 1938; Herrick, 1956; Keller, 1941; Roufs, 1972).

During dominance, there is an increase in threshold for the amblyopic eye relative to the normal or nonamblyopic eye (for both monoptic and dichoptic viewing). This elevation of threshold is constant for all durations and ranges from 0.3 to $0.6 \log$ units between observers (i.e., there is no change to $t_{\mathrm{c}}$, but there is an increase in $I_{\mathrm{c}}$ ). Grosvenor (1957) reported an elevated threshold for wide bar stimuli in the fovea of the amblyopic eye of $0.3-0.6 \log$ units at different background intensities. Grosvenor also found the increase in threshold to be constant at three durations ( 10 , 50 , and $200 \mathrm{msec}$ ) for all but the brightest background intensity (estimated to be 3,000 td, assuming a 4-mm pupil). An increase in threshold (about $0.5 \log$ units) for large area foveal stimuli in the amblyopic eye has also been reported by Flynn (1967).

Harwerth and Levi (1978b) and Loshin and Jones (1982) studied contrast sensitivity as a function of exposure duration for specific spatial frequencies. They found that $t_{\mathrm{c}}$ varied as a function of the spatial frequency, with longer integration times for higher spatial frequencies. The amblyopic eyes had normal $t_{\mathrm{c}}$ values and normal summation slopes ( 0.7 for gratings) at low spatial frequencies. However, at high spatial frequencies, $t_{c}$ values were longer, and the summation slopes were shallower (slopes as flat as 0.4 ). They also found that $I_{\mathrm{c}}$ varied as a function of spatial frequency: At low spatial frequencies, there was a small increase in $I_{c}$ (about $0.1 \mathrm{log}$ units for 0.5 cycles per degree), and, at high spatial frequencies, there was a larger increase (about $0.5 \mathrm{log}$ units for 8.0 cycles per degree). These results suggest that, had we used a high spatial frequency stimulus, we might have reached different conclusions.

Threshold-duration functions obtained for the amblyopic eye with monocular viewing are similar to those obtained with the dominance condition. This may seem surprising, considering that visual acuity in the amblyopic eye is reduced further when measured with the nonamblyopic eye open (Pugh, 1954; Schor, Terrell, \& Peterson, 1976; von Noorden \& Leffler, 1966). However, visual acuity and increment thresholds are very different tasks. This finding implies that (for Observers G.N. and S.D.) under these conditions, there is little or no inhibitory effect exerted on the amblyopic eye from the nonamblyopic eye.

During induced anisometropic suppression in the normal eye or in the nonamblyopic eye, there is an increase in threshold that is constant for all durations (i.e., no change to $t_{\mathrm{c}}$, an increase in $I_{\mathrm{c}}$ ). The magnitude of the threshold elevation ranges from 0.4 to $0.6 \log$ units between observers.

Induced anisometropic suppression in the amblyopic eye causes a threshold elevation of $0.1-0.5 \log$ units. This elevation is again constant for all durations. For most observers, threshold is elevated by $0.1-0.3 \log$ units. One observer showed a threshold elevation of $0.5 \log$ units. The threshold elevation caused by induced anisometropic suppression in the amblyopic eye is always less than that induced in the nonamblyopic eye or in normal eyes. This result supports that of Holopigian, Blake, and Greenwald (1988), who found that the degree of amblyopia and the depth of suppression were inversely related, which implies that amblyopic eyes do not have a need for strong suppression.

Are induced anisometropic suppression and binocular rivalry suppression related? It seems sensible to suggest that these conditions may be related, because they both occur in response to conflicting monocular visual input and 
they both result in the functional loss of vision from one eye. This experiment provides two lines of evidence that suggest that they may be related. First, the threshold elevation during anisometropic suppression is equal for all durations, suggesting nonselectivity - a feature of binocular rivalry suppression (Blake \& Fox, 1974; Fox \& Check, 1966, 1968; Holopigian, 1989; Wales \& Fox, 1970). Second, the threshold elevation is of similar magnitude to that reported for binocular rivalry suppression (Wales \& Fox, 1970).

Is induced anisometropic suppression related to anisometropic amblyopia? It seems reasonable to suggest that these conditions are related, because the instigating circumstances (dissimilar monocular images) are comparable in the two cases. A similar line of argument that is used to relate anisometropic suppression to binocular rivalry can be used here. In the amblyopic eye, there is a threshold elevation that is nonselective for duration. This result is qualitatively (nonselectivity) and quantitatively (magnitude of the threshold elevation) similar to the result for induced anisometropic suppression in normal eyes or in the nonamblyopic eye. However, Blake (1989) pointed out that binocular rivalry suppression in normal individuals usually involves alternations in dominance between the two eyes, whereas amblyopia is unilateral. Holopigian et al. (1988) suggest that binocular rivalry suppression and clinical suppression are fundamentally different, because clinical suppression can occur while observers view identically orientated gratings presented dichoptically, whereas binocular rivalry occurs when observers view orthogonally orientated gratings. The magnitude of suppression for clinical suppressors was generally greater than that during binocular rivalry suppression and was consistent within observers, regardless of the orientation of the dichoptic stimuli. Therefore, it seems unlikely that anisometropic amblyopia is a form of anisometropic suppression. However, this does not rule out the possibility that anisometropic suppression leads to anisometropic amblyopia during development.

It is informative to apply Blake's (1989) model of binocular rivalry to the anisometropic visual system during development. Early in development, ocular dominance distributions are almost normal: Binocular neurons outnumber monocular neurons, and monocular neurons innervated exclusively by the left eye are no more numerous than monocular neurons innervated exclusively by the right eye (Hubel, Wiesel, \& Le Vay, 1975). From Proposition 6 of Blake's model (the strength of inhibition is based on the pool of monocular neurons receiving inhibition), unilateral defocus (anisometropia) will result in a moderate amount of suppression. This suppression will inhibit the development of monocular neurons innervated by the defocused eye, because they will receive reduced input. It will also inhibit the development of binocular neurons. The result will be an ocular dominance distribution with a reduction in binocular neurons and a shift in the proportion of monocular neurons in favor of the eye with no defocus. It seems reasonable that inhibition of development will be greatest in neurons that are most responsive to stimulus characteristics that are suppressed. Therefore, anomalies of the amblyopic visual system should reflect anomalies of the normal visual system during suppression (Hubel et al., 1975). This reasoning relies on the presence of suppression mechanisms early in human visual development. Birch, Shimojo, and Held (1985) and Shimojo, Bauer, O'Connell, and Held (1986) have shown binocular rivalry to be present at about 4 months of age in humans (preferential looking); therefore, it is likely that other suppression mechanisms are also present.

In conclusion, the major finding from this study is that reaction time versus intensity functions are shape-invariant, which is consistent with the notion that a single mechanism mediates detection under these experimental conditions. This also means that, although reaction times are longer during induced anisometropic suppression or in anisometropic amblyopia, they are equal if contrast is normalized to equate threshold. Temporal summation, as assessed by the critical duration, does not change for any of these conditions. We also show that anisometropic amblyopia and induced anisometropic suppression behave similarly. They are probably not the same condition, although anisometropic amblyopia may be a developmental consequence of anisometropic suppression early in life.

\section{REFERENCES}

Birch, E. E., Shimojo. S., \& Held, R. (1985). Preferential looking assessment of fusion and stereopsis in infants ages 1-6 months. Investigative Ophthalmology \& Vision Science, 26, 366-370.

BL ACKWELL, H. R. (1946). Contrast threshold of the human eye. Journal of the Optical Society of America, 36, 624-643.

BLAKE, R. (1989). A neural theory of binocular rivalry. Physiological Review, 96, 145-167.

BLAKE, R., \& Fox, R. (1974). Binocular rivalry suppression: Insensitive to spatial frequency and orientation change. Vision Research, 14, 687-692.

BURIAN, H. M., \& VON NOORDEN, G. K. (1974). Binocular vision and ocular motility: Theory and management of strabismus. St Louis: Mosby.

Ciuffreda, K. J., LeVi, D. M., \& Selenow, A. (1991). Amblyopia: Basic and clinical aspects. Boston: Butterworth-Heinemann.

DUKe-Elder, S., \& WyBAR, K. (1973). Systems of ophthalmology (Vol. 6). London: Kimpton.

FLYNN. I. T. (1967). Spatial summation in amblyopia. Archives of Ophthalmology, 78, 470-474.

Fox, R., \& CHeCk, R. (1966). Binocular fusion: A test of the suppression theory. Perception \& Psychophysics, 1, 331-334.

Fox, R., \& Check, R. (1968). Detection of motion during binocular rivalry suppression. Journal of Experimental Psychology, 78, 388-395.

Fox, R., TODD, S., \& BetTINGER, L. A. (1975). Optokinetic nystagmus as an objective indicator of binocular rivalry. Vision Research, 15, 849-853.

Fuortes, M. G. F., Gunkel, R. D., \& Rushton, W. A. H. (1961). Increment thresholds in a subject deficient in cone vision. Journal of Physiology, 156, 179-192.

Graham, C. H., \& KeMP, E. H. (1938). Brightness discrimination as a function of the duration of the increment in intensity. Journal of General Physiology, 21, 635-650.

GrosvenOR, T. (1957). The effects of duration and background luminance upon the brightness discrimination of an amblyope. American Journal of Optometry \& Physiological Optics, 34, 634-663.

HAMASAKI, D. I., \& FLYNN, J. T. (1981). Amblyopic eyes have longer reaction times. Investigative Ophthalmology \& Vision Science, 21, 846-853. 
Harwerth, R. S., Boltz, R. L., \& Smith, E. L., III (1980). Psychophysical evidence for sustained and transient channels in the monkey visual system. Vision Research, 20, 15-22.

HaRwerTh, R. S., \& LeVI, D. M. (1978a). Reaction time as a measure of suprathreshold grating detection. Vision Research, 18, 1579-1586.

HARWERTH, R. S., \& LEVI, D. M. (1978b). A sensory mechanism for amblyopia: Psychophysical studies. American Journal of Optometry \& Physiological Optics, 55, 151-162.

HERRICK, R. M. (1956). Foveal luminance discrimination as a function of the duration of the decrement or increment in luminance. Journal of Comparative Physiology \& Psychology, 49, 437-443.

HoLOPIGIAN, K. (1989). Clinical suppression and binocular rivalry suppression: The effects of stimulus strength on the depth of suppression. Vision Research, 29, 1325-1333.

Holopigian, K., Blake, R., \& Greenwald, M. (1988). Clinical suppression and amblyopia. Investigative Ophthalmology \& Vision Science, 29, 444-451.

HuBEL, D. H., WiESEL, T. N., \& LE VAY, S. (1975). Functional architecture of area 17 in normal and monocularly deprived macaque monkeys. Cold Spring Harbor Symposium Proceedings, 40, 581-589.

HUMPHRISS, D. (1959). Refraction by the Humphriss immediate contrast method. Optician, 138, 372-373.

HuMPhriss, D. (1960). Refraction by immediate contrast. Optician, 139, 159-160.

HuMPhrISs, D. (1963). The refraction of binocular vision. Ophthalmic Optician, 3, 987-1001.

HUMPHRISS, D. (1982). The psychological septum: An investigation into its function. American Journal of Optometry \& Physiological Optics, 59, 639-641.

Humphriss, D., \& WoOdRUFF, E. (1962). Refraction by immediate contrast. British Journal of Physiological Optics, 19, 15-20.

Kalloniatis, M., \& HaRWeRTH, R. S. (1990). Spectral sensitivity and adaptation characteristics of cone mechanisms under white-light adaptation. Journal of the Optical Society of America A, 7, 1912-1928.

Kalloniatis, M., \& HARWERTH, R. S. (1991). Effects of chromatic adaptation on opponent interactions in monkey increment-threshold spectral sensitivity functions. Journal of the Optical Society of America $A, 8,1818-1831$.

KELLER, M. (1941). The relation between the critical duration and intensity in brightness discrimination. Journal of Experimental Psychology, 28, 407-418.

Kohfeld, D. L., Santee, J. L., \& Wallace, N. D. (1981a). Loudness and reaction time: I. Perception \& Psychophysics, 29, 535-549.

KoHFELD, D. L., SANTEE, J. L., \& WALLACE, N. D. (1981b). Loudness and reaction time: II. Identification of detection components at different intensities and frequencies. Perception \& Psychophysics, 29, 550-562.

LEHKY, S. R. (1988). An astable multivibrator model of binocular rivalry. Perception, 17, 215-228.

LEVELT, W. J. M. (1965). On binocular rivalry. Soesterberg, The Netherlands: Institute for Perception RVO-TNO.

LEVI, D. M., HARWERTH, R. S., \& MANNY, R. E. (1979). Suprathreshold spatial frequency detection and binocular interaction in strabismic and anisometropic amblyopia. Investigative Ophthalmology \& Vision Science, 18, 714-725.

LOSHIN, D. S., \& JONES, R. (1982). Contrast sensitivity as a function of exposure duration in the amblyopic visual system. American Journal of Optometry \& Physiological Optics, 59, 561-567.

LUCE, R. D. (1986). Response times: Their role in inferring elementary mental organization. New York: Oxford University Press.

Mackenson, G. (1958). Reaktionszeitmessungen bei Amblyopie [Reaction time in amblyopia]. Graefe's Archives of Clinical \& Experimental Ophthalmology, 159, 636-648.

MANSFIELD, R. J. W. (1973). Latency functions in human vision. Vision Research, 13, 2219-2234.
MILLER, E. F. (1955). The nature and cause of impaired vision in the amblyopic eye of a squinter. American Journal of Optometry, 32, 10-18.

NygaARD, R. W., \& FRUMKEs, T. E. (1982). Calibration of the retinal illuminance provided by Maxwellian views. Vision Research, 22, 433-434.

O'SHEA, R. P. (1987). Chronometric analysis supports fusion rather than suppression theory of binocular vision. Vision Research, 27, 781-791.

O'ShEA, R. P., \& CRASSINI, B. (1981). The sensitivity of binocular rivalry suppression to changes in orientation assessed by reaction-time and forced-choice techniques. Perception, 10, 283-293.

Piéron, H. (1914). Recherches sur les lois de variation des temps de latence sensorielle en fonction des intensités excitatrices [Research into the variation of sensory latency as a function of intensity]. L'Année Physiologique, 20, 17-96.

PlÉRON, H. (1920). Nouvelles recherches sur l'analyse du temps de latence sensorielle et sur la loi qui relie le temps à l'intensité d'excitation [New research on the analysis of sensory latency and how it varies with intensity]. L'Année Physiologique, 22, 58-142.

Pugh, M. (1954). Foveal vision in amblyopia. British Journal of Ophthalmology, 38, 321-331.

RouFs, J. A. J. (1972). Dynamic properties of vision: I. Experimental relationships between flicker and flash thresholds. Vision Research, 12, 261-278.

Schor, C. M., Landsman, L., \& Erickson, P. (1987). Ocular dominance and the interocular suppression of blur in monovision. American Journal of Optometry \& Physiological Optics, 64, 723-730.

Schor, C. M., Terrell, M., \& Peterson, D. (1976). Contour interaction and temporal masking in strabismus and amblyopia. American Journal of Optometry \& Physiological Optics, 53, 217-223.

Shimojo, S., Bauer, J., O'ConNell, K. M., \& Held, R. (1986). Prestereopic binocular fusion in infants. Vision Research, 26, 501-510.

SIMPSON, T. (1991). The suppression effect of simulated anisometropia. Ophthalmic \& Physiological Optics, 11, 350-358.

Simpson, T. (1992). Monocular acuity in the presence and absence of fusion. Optometry \& Vision Science, 69, 405-410.

Simpson, T., Smith, E. L., III, HaRWerth, R. S., \& Kalloniatis, M. (1990). Spectral sensitivity of induced anisometropic suppression. Investigative Ophthalmology \& Vision Science (Suppl.), $31,94$.

UENO, T. (1977). Reaction time as a measure of temporal summation at suprathreshold levels. Vision Research, 17, 227-232.

UENo, T. (1978). Temporal summation in human vision: Simple reaction time measurements. Perception \& Psychophysics, 23, 43-50.

VON NOORDEN, G. K. (1961). Reaction time in normal and amblyopic eyes. Archives of Ophthalmology, 66, 695-701.

von Nooroen, G. K., \& LefFler, M. B. (1966). Visual acuity in strabismus amblyopia under monocular and binocular conditions. Archives of Ophthalmology, 76, 172-177.

WALES, R., \& Fox, R. (1970). Increment detection thresholds during binocular rivalry suppression. Perception \& Psychophysics, 8, 90-94.

WALKER, P. (1975). Stochastic properties of binocular rivalry alternations. Perception \& Psychophysics, 18, 467-473.

WATSON, A. B. (1986). Temporal sensitivity. In K. R. Boff, L. Kaufman, \& J. P. Thomas (Eds.), Handbook of perception and human performance (pp. 6-1 to 6-43). New York: Wiley.

WEBER, A. I. (1988). Ricco's area and resolution in the human visual system. Unpublished master's thesis, University of Houston.

WOLFE, J. M., \& OWENS, D. A. (1979). Evidence for separable binucular processes differentially affected by artificially induced anisometropia. American Journal of Optometry \& Physiological Optics, 56, 279-284.

(Manuscript received November 26, 1996; revision accepted for publication April 23, 1997.) 